DOI: $10.17516 / 1997-1397-2021-14-2-144-149$

УДК 621.373 .826

\title{
Simulation of Broadening the Second Harmonic Spectrum in KDP by Chirp Pulse Pumping
}

\author{
Sergey V. Alekseev* \\ Valery F. Losev ${ }^{\dagger}$ \\ Institute of High Current Electronics SB RAS \\ Tomsk, Russian Federation \\ Yakov V. Grudtsyn \\ Andrey V. Koribut ${ }^{\S}$ \\ P. N. Lebedev Physical Institute of RAS \\ Moscow, Russian Federation \\ Vyacheslav A. Trofimov \\ South China University of Technology \\ Guangzhou, China
}

Received 10.08.2020, received in revised form 10.09.2020, accepted 20.11.2020

\begin{abstract}
The conditions of broadening the second harmonic spectrum in KDP upon pumping by a negatively chirped pulse of fundamental frequency with central wavelength of $950 \mathrm{~nm}$ are analyzed numerically. It is shown that broadening of the spectrum $(\mathrm{K}=1.4)$ is mainly limited by difference in group velocities of radiation pulse between first and second harmonics. The article is based on materials of the report at the first All-Russian scientific conference with international participation "YENISEI PHOTONICS - 2020".

Keywords: femtosecond laser pulse, second harmonic, spectrum, efficiency, pulse duration.

Citation: S.V.Alekseev, V.F. Losev, Ya.V.Grudtsyn, A.V. Koribut, V.A. Trofimov, Simulation of Broadening the Second Harmonic Spectrum in KDP by Chirp Pulse Pumping, J. Sib. Fed. Univ. Math. Phys., 2021, 14(2), 144-149. DOI: 10.17516/1997-1397-2021-14-2-144-149.
\end{abstract}

\section{Introduction}

Currently, all powerful femtosecond laser systems operate in the infrared spectrum range (0.8-1 microns). The expansion of their spectral range will make it possible to push the boundaries of the application fields of these systems and, in some cases, will help to increase the interaction efficiency of femtosecond radiation pulses with matter. The way to advance into the visible region of the spectrum due to the generation of the second harmonic $(\mathrm{SH})$ during the conversion of infrared (IR) radiation in a nonlinear crystal is known. However, the possibility of converting high peak power IR radiation into $\mathrm{SH}$ is limited by the technological difficulties of manufacturing thin (less than $1 \mathrm{~mm}$ thickness) nonlinear crystals with a sufficiently large diameter $(20 \mathrm{~cm}$ or more), and the low quality of SH radiation due to phase self-modulation, cross-modulation, Kerr self-focusing, and deep spectrum modulation in a nonlinear crystal.

\footnotetext{
*sergey100@sibmail.com

$\dagger$ †osev@ogl.hcei.tsc.ru

$\ddagger$ grudcynyv@lebedev.ru

§koributav@lebedev.ru

ฯtrofimov@scut.edu.cn

(C) Siberian Federal University. All rights reserved
} 
In the last decade, an alternative way of creating multi-terawatt laser systems has been developed in HCEI SB RAS, Tomsk. It is based on the use of a solid-state femtosecond laser complex and a photodissociation $\mathrm{XeF}(\mathrm{C}-\mathrm{A})$ amplifier with a gas active medium $[1,2]$. The advantages of this hybrid scheme are the low optical nonlinearity of the gas active medium, the visible radiation range $(475 \mathrm{~nm})$, the ability to scale the gas amplifier, the achievement of high contrast due to the conversion of radiation in a nonlinear crystal and a low gain in the gas medium. In 2012, the THL-100 laser system produced a record peak power of $14 \mathrm{TW}$ for the visible spectrum $[1,2]$. In recent years, research has been conducted to increase this power.

An attractive method for increasing peak power is to reduce the duration of a transform limited radiation pulse without increasing its energy. To reduce the pulse duration, it is necessary to increase the width of its spectrum. For this purpose, phase modulation is usually used when a femtosecond pulse passes through gaseous or solid media. Since our system uses a non-linear crystal (KDP), it suggests organizing a broadening of the spectrum with a subsequent reduction in the duration of the SH pulse directly in the non-linear crystal itself [3]. Unfortunately, this method of broadening is poorly studied in the literature, and we have found only one work devoted to this [4], which showed the possibility of increasing the spectrum width in a nonlinear $\mathrm{BBO}$ crystal and reducing the duration of the $\mathrm{SH}$ pulse with a wavelength of $400 \mathrm{~nm}$ to two times.

In our opinion, additional theoretical and experimental studies are required to find optimal conditions for broadening the SH spectrum and obtaining information about the physical processes responsible for this.

In this regard the present work is devoted to study of conditions of SH generation upon pumping a nonlinear crystal at a wavelength of $950 \mathrm{~nm}$ with transform limited and chirped pulses in order to determine the processes that affect the broadening of the SH spectrum.

\section{Instrumentation and techniques}

The experiments were carried out on a Ti:Sa femtosecond complex, which is a front-end for THL-100 multi-terawatt laser system. The complex consists of a master oscillator, stretcher, regenerative and two multi-pass amplifiers, compressor on diffraction gratings, and a SH generator ( $2 \mathrm{~mm}, \mathrm{KDP}$ crystal). The complex operates at a central wavelength of $950 \mathrm{~nm}$ with $50-70 \mathrm{fs}$ pulse duration, the radiation pulse had $10 \mathrm{~mJ}$ energy. The transform limited radiation pulse with 60 fs duration or negatively chirped pulse with $700 \mathrm{fs}$ duration were used. The pulse was chirped in the compressor by increasing the distance between the gratings. After compressor, the Gaussian fundamental frequency beam had $16 \mathrm{~mm}$ diameter at $1 / \mathrm{e} 2$ level. In front of the KDP the beam radius was reduced by factor of two in mirror telescope. The transform limited duration of SH chirped pulse was obtained using compression in glass block.

To measure the energy of laser radiation Gentec and OPHIR meters were used. The emission spectra were measured using ASP150C spectrometers (Avesta-project) and Ocean Optics HR4000 (200-1100 nm, $0.7 \mathrm{~nm})$.

The simulation model allows analyzing the influence of such factors as: difference between group velocities of the first and second harmonics, self-phase modulation, and dispersive spreading of radiation pulses. The model was based on solving a system of nonlinear Schrodinger equations for the first and second harmonics in slowly varying wave approximation [5]. The system of equations describing the process of second harmonic generation in the approximation of slowly varying amplitudes has the following form:

$$
\frac{\partial A_{1}}{\partial z}+i D_{1} \frac{\partial^{2} A_{1}}{\partial \eta^{2}}+i D_{\perp} \Delta_{\perp} A_{1}+i \gamma A_{1}^{*} A_{2} e^{-i \Delta k x}+i \alpha_{1} A_{1}\left(\left|A_{1}\right|^{2}+2\left|A_{2}\right|^{2}\right)=0, \quad 0<z<L_{z}
$$




$$
\begin{gathered}
\frac{\partial A_{2}}{\partial z}+\nu \frac{\partial A_{2}}{\partial \eta}+i D_{2} \frac{\partial^{2} A_{2}}{\partial \eta^{2}}+i D_{\perp} \Delta_{\perp} A_{2}+i \gamma A_{1}^{2} e^{i \Delta k x}+i \alpha_{2} A_{1}\left(2\left|A_{1}\right|^{2}+\left|A_{2}\right|^{2}\right)=0, \\
\alpha_{2}=2 \alpha_{1}=2 \alpha, \\
\Delta_{\perp}=\frac{1}{r} \frac{\partial}{\partial r}\left(r \frac{\partial}{\partial r}\right),
\end{gathered}
$$

where $\eta$ is the dimensionless time in the main coordinate system accompanying the pulse, $\mathrm{z}$ is the longitudinal coordinate, $D_{j} \sim-0.5 \frac{\partial^{2} k}{\partial \omega^{2}}$ are the coefficients characterizing the second-order dispersion, $\gamma$ is the coefficient of nonlinear coupling of the interacting waves, $\Delta k=k_{2}-2 k_{1}$ is the detuning of the wave numbers. The complex amplitudes of the harmonics normalized to the maximum amplitude of the first harmonic are taken in the initial section of medium $(z=0)$. The parameter $\nu$ is proportional to the difference between the reciprocal values of the group velocities of the second harmonic waves and the fundamental frequency, $L_{z}$ is the length of the nonlinear medium.

The initial distribution of first harmonic amplitude is modeled by a Gaussian pulse taking into account the normalization and chirp parameter $C$ :

$$
A(\eta, r)=\exp \left(-(1+i C)\left(\left(\eta-L_{t} / 2\right) / \tau\right)^{2} / 2-\left(r / r_{0}\right)^{2} / 2\right) .
$$

Such chirped pulse can be compressed to transform limited one with the duration $\sqrt{1+C^{2}}$ shorter than the original. The coefficient $\gamma$ was determined from experimental data, $\nu$ and $D_{j}$ were calculated using the Selmeyer formula by differentiating $n$ with respect to the wavelength [6]:

$$
\begin{aligned}
& n_{0}^{2}=2.259276+\frac{13.00522 \lambda^{2}}{\lambda^{2}-400}+\frac{0.01008956}{\lambda^{2}-0.0129426} \\
& n_{e}^{2}=2.132668+\frac{3.2279924 \lambda^{2}}{\lambda^{2}-400}+\frac{0.008637494}{\lambda^{2}-0.0122810} .
\end{aligned}
$$

For the analysis, we took conditions close to the experimental ones, namely: wavelength of the first harmonic was $950 \mathrm{~nm}$, duration of the transform limited pulse was $60 \mathrm{fs}$ and 700 fs for chirped pulse, and $10 \mathrm{~mJ}$ energy. The conversion to the second harmonic was simulated in a KDP crystal of 1st phase-matching type with thickness of $2 \mathrm{~mm}$.

The phase matching angle $\left(\theta=41.6^{\circ}\right)$ was found from the dependence $n_{o}(950 \mathrm{~nm})=$ $=n_{e}(475 \mathrm{~nm})$, and the spectral phase matching width $(\Delta \lambda=8.5 \mathrm{~nm})$ from the condition $\sin c^{2}\left(\frac{\Delta k L}{2}\right)=0.5$, where $\Delta k=k(950+\Delta \lambda)-k(475+\Delta \lambda / 2)$. Since the total spectrum width is $18 \mathrm{~nm}$, the narrowing effect due to the finite phase matching width can be neglected during conversion.

\section{Results and discussion}

To clarify our experimental data, Fig. 1 shows the spectral composition of second harmonic radiation for transform limited (1) and chirped (2) pulses. It can be seen that when a chirped pulse is used, approximately one and a half times, rather than a twofold broadening of the spectrum, as in [1], is observed.

The task of our calculations was to find the reasons for this behavior of SH spectrum and verify the analytical conclusion [1] taking into account all main processes accompanying the second harmonic generation process: dispersive spreading of pulses, runaway of pulses in the crystal thickness due to difference in group velocities, effect of Kerr nonlinearity, etc. Let us 


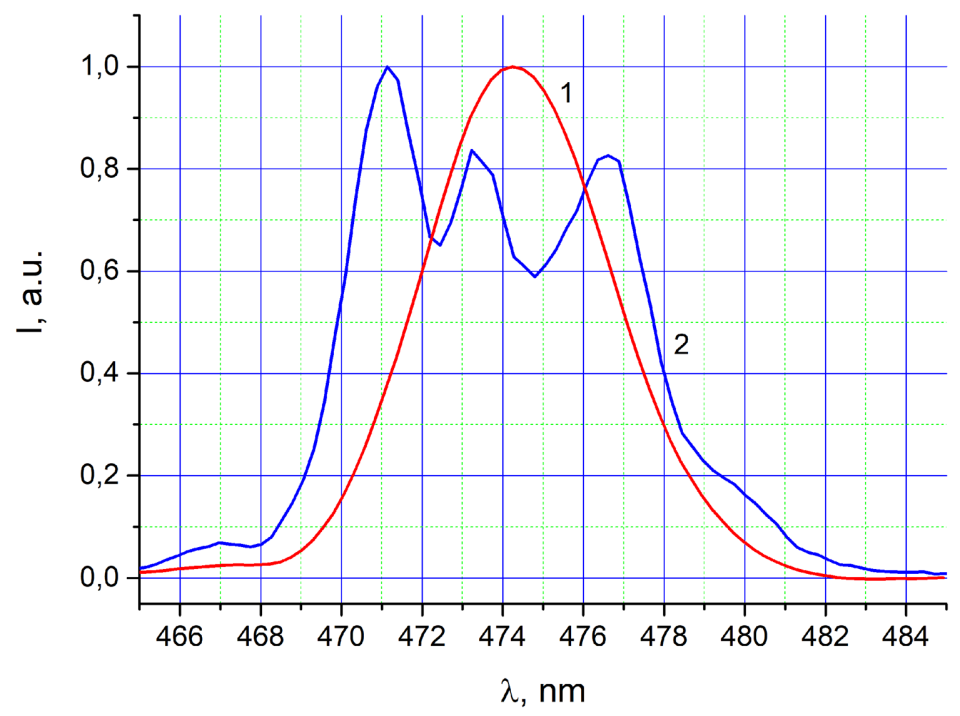

Fig. 1. Spectra of SH radiation pulses: 1 - transform limited pulse, 2 - negatively chirped pulse

begin with analysis of the results of [1], where the task of chirped pulse converting was carried out analytically neglecting the effects of dispersion and nonlinearity. In the case of transform limited pulse (the pump envelope has the form $\exp \left(-t^{2}\right)$ ) and with a low conversion efficiency in $\mathrm{SH}$, its envelope will look like $\exp \left(-2 t^{2}\right)$. This means that the pulse duration will be reduced by $\sqrt{2}$ times. Accordingly the spectral width should increase (up to $7.8 \mathrm{~nm}$ with pulse duration at the first harmonic of $60 \mathrm{fs}$ ). With increase of conversion efficiency the pulse at second harmonic approaches to the pulse of first harmonic $\exp \left(-t^{2}\right)$, that is, its duration increases and the spectrum narrows. If a chirped pulse $\exp \left(-(1+i C) t^{2}\right)$ is converted, then with low conversion efficiency for SH pulse the expression $\exp \left(-(1+i C)(\sqrt{2} t)^{2}\right)$ will be valid. With increasing of efficiency, this expression tends to $\exp \left(-(1+2 i C) t^{2}\right)$. A chirped pulse $\exp \left(-(1+i C) t^{2}\right)$ with a chirp parameter $C$ can be compressed to a transform limited one, while shortening the duration in $\sqrt{1+C^{2}}$ times.

From this it can be seen that the ratio of minimum pulse duration at first harmonic to second is for low conversion efficiency $\frac{1}{\sqrt{1+C^{2}}} / \frac{1}{\sqrt{2} \sqrt{1+C^{2}}}=\sqrt{2}$, and for high efficiency it can reach 2 , according to the relation $d \frac{1}{\sqrt{1+C^{2}}} / \frac{1}{\sqrt{1+4 C^{2}}}$. Thus, for a chirped pulse, the opposite process takes place - the spectrum width increases with an increase in the conversion efficiency.

Simulations showed that self-phase modulation and dispersive spreading of pulses insignificantly effect on SH emission spectrum broadening. In Fig. 2 shows the calculation results showing the dependence of SH radiation spectrum width on conversion efficiency for negatively chirped and transform limited pulses.

It can be seen that general trend remains with the results obtained in [1], where approximate consideration was used, namely: with increase of conversion efficiency the spectral width of second harmonic increases in case of first harmonic chirped pulse, and decreases for transform limited pulse. Analysis of the factors influencing broadening of SH spectrum in simulation showed that the main factor limiting the broadening of SH spectrum during the transformation of chirped pulse was the difference in group velocities. The dispersion of group velocities limited the broadening at level $K=\Delta \lambda_{c h} / \Delta \lambda=1.1$, where $\Delta \lambda_{c h}$ is the width of the second harmonic spectrum upon pumping with chirped pulse, and $\Delta \lambda-$ upon pumping by transform limited 


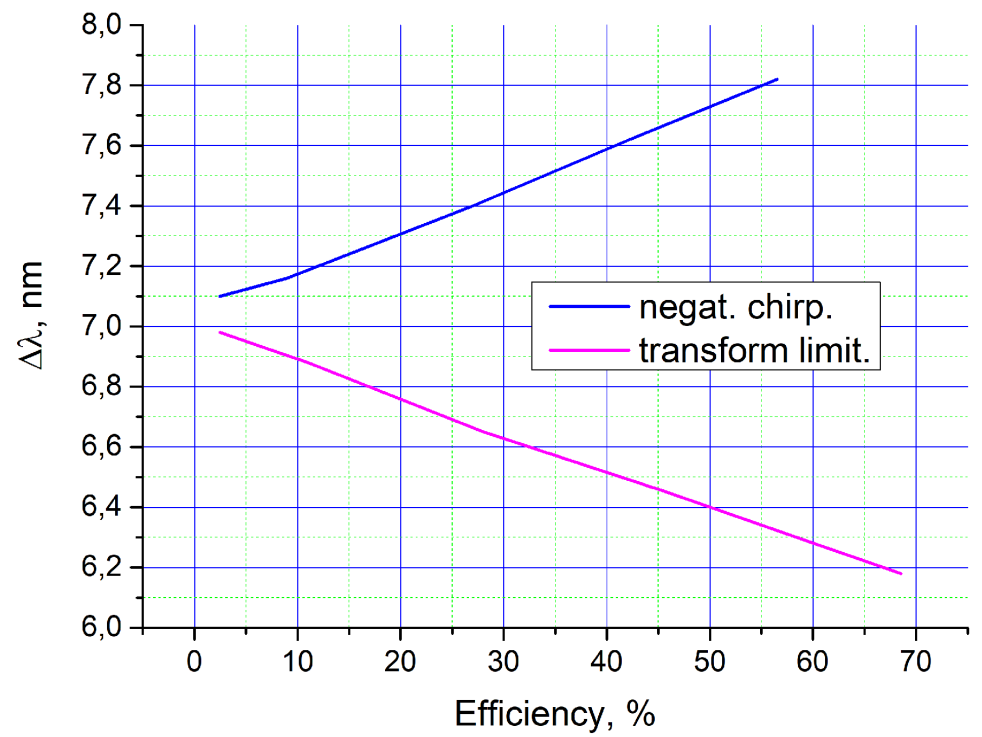

Fig. 2. Dependence of the width of SH radiation spectrum on conversion efficiency for $2 \mathrm{~mm}$ KDP crystal

pulse. This is less than theoretically possible $K=1.4$, which can be obtained with low conversion efficiency. Nevertheless, taking into account the decrease of second harmonic spectrum width upon the transformation of transform limited first-harmonic pulse with increase of conversion efficiency, in the calculation, the spectral width for chirped pulse at high conversion efficiency was 1.3 times greater than conversion of transform limited pulse. This calculated value is close to that obtained experimentally (Fig. 1).

\section{Conclusion}

Thus, in result of numerical simulation of second harmonic spectrum broadening in KDP upon pumping by negatively chirped pulse of fundamental frequency with central wavelength of 950 $\mathrm{nm}$ and pulse duration of $700 \mathrm{fs}$, a fairly good agreement was obtained between the spectrum broadening $(K=1.3)$ and experimental result $(K=1.4)$. It was shown that SH spectrum broadening is mainly limited by the difference in group velocities of radiation pulse of first and second harmonics.

The experimental study was carried out with the financial support of Russian Foundation for Basic Research within the framework of scientific project no. 18-08-00383 and numerical calculations with financial support of Russian Science Foundation for no. 19-11-00113.

\section{References}

[1] S.V.Alekseev, A.I.Aristov, N.G.Ivanov, et all., Multi-terawatt femtosecond laser system of visible range based on a photochemical $\mathrm{XeF}(\mathrm{C}-\mathrm{A})$ amplifier, Laser and Particle Beams, 31(2013), 17-21. DOI: 10.1017/S0263034612000870

[2] S.V.Alekseev, A.I.Aristov, Ya.V.Grudtsyn, et all., Visible-range hybrid femtosecond systems based on a $\mathrm{XeF}(\mathrm{C}-\mathrm{A})$ amplifier: state of the art and prospects, Quantum Electronics, 43(2013), 190-200. DOI: 10.1070/QE2013v043n03ABEH015096 
[3] S.V.Alekseev, M.V.Ivanov, N.G.Ivanov, V.F.Losev, Influence of chirp of the fundamental frequency pulse radiation on the spectral-time characteristics of second harmonic , Izvestiya vuzov. Fizika, 12(2019), 151-155 (in Russian). DOI: 10.17223/00213411/62/12/151

[4] N.V.Didenko, A.V.Konyashchenko, L.L.Losev, et all., Femtosecond pulse compression based on second harmonic generation from a frequency chirped pulse, Optics Communications, 282(2009), 997-999.

[5] V.A.Trofimov, T.M.Lysak, Highly efficient SHG of a sequence of laser pulses with a random peak intensity and duration, Optics and Spectroscopy, 107(2009), 399-406.

DOI: $10.1134 /$ S0030400X0909015X

[6] V.G.Dmitriev, G.G.Gurzadyan, D.N.Nikogosyan, Handbook of Nonlinear Optical Crystals, Third Revision, Springer series in optical sciences, Springer Berlin, 64(1999), 78-85.

\title{
Анализ условий уширения спектра второй гармоники в КДП при накачке чирпированным импульсом основной частоты
}

\author{
Сергей В. Алексеев \\ Валерий $\Phi$. Лосев \\ Институт сильноточной электроники СО РАН \\ Томск, Российская Федерация \\ Яков В. Грудцын \\ Андрей В. Корибут \\ Физический институт имени П. Н. Лебедева \\ Москва, Российская Федерация \\ Вячеслав А. Трофимов \\ Южно-Китайский университет технологий \\ Гуанчжоу, Китай
}

\begin{abstract}
Аннотация. Численно анализируются условия уширения спектра второй гармоники в КДП при накачке отрицательно чирпированным импульсом основной частоты с центральной длиной волны 950 нм. Показывается, что величина уширения спектра $(\mathrm{K}=1.4)$ в основном ограничивается разностью групповых скоростей импульса излучения первой и второй гармоник. Статья подготовлена по материалам доклада на Первой Всероссийской научной конференции с международным участием «ЕНИСЕЙСКАЯ ФОТОНИКА - 2020».
\end{abstract}

Ключевые слова: фемтосекундный лазерный импульс, вторая гармоника, спектр, эффективность, длительность импульса. 